\title{
Aproximación preliminar a la estructura de una colonia de Natalus tumidirostris (Natalidae) durante la época de lluvia en la cueva Macaregua, Santander, Colombia
}

\author{
Preliminary approach to the structure of a colony of \\ Natalus tumidirostris (Natalidae) during the rainy season at the \\ Macaregua cave, Santander, Colombia
}

\author{
Ana Lucía Rueda-Ardila*, Jairo Pérez-Torres*
}

\begin{abstract}
Resumen
Introducción: Se describen el tamaño poblacional y aspectos reproductivos de una colonia de Natalus tumidirostris durante tres muestreos en la época de lluvia del año 2013, en la cueva Macaregua (6³9'36.2" N y $73^{\circ} 0.6^{\prime} 32.3^{\prime \prime}$ W) en el municipio de Curití, Santander, Colombia. Objetivo: Estimar la proporción de sexos, categorías de edad, estado reproductivo y tamaño poblacional de una colonia de $N$. tumidirostris. Metodología: Se capturaron individuos de N. tumidirostris con una trampa arpa artesanal, red de niebla modificada y captura manual. Resultados: Se capturaron en total 274 individuos de los cuales 14 fueron recapturas. La proporción de sexos durante los muestreos fueron de 7:1 (junio), 1:1 (agosto) y 3:1 (septiembre). La pirámide poblacional fue de forma invertida y se reportó la presencia de una guardería en el mes de abril. En cuanto al estado reproductivo de las hembras, algunas estaban inactivas (42\%) y otras (58\%) preparando para eventos reproductivos (proestro). No se observó actividad reproductiva en los machos. El tamaño de la colonia está en un rango entre 1941 y 3279 individuos. Conclusión: Para los meses de estudio no se presentó segregación sexual, aunque sí hubo variaciones en la proporción de sexos. La presencia de una guardería y la descripción de la condición reproductiva de las hembras indican que el período de reproducción para esta especie se da en los primeros meses del año, entre enero y febrero.
\end{abstract}

Palabras clave: Categoría de edad, Estado reproductivo, Chiroptera, Natalidae, Tamaño poblacional.

\begin{abstract}
Introduction: We describe the population size and reproductive aspects of a colony of Natalus tumidirostris during the rainy season of 2013, in the Macaregua cave (6 $6^{\circ} 39^{\prime} 36.2$ " $\mathrm{N}$ and $73^{\circ} 0.6^{\prime} 32.3^{\prime \prime} \mathrm{W}$ ), Curití, Santander, Colombia. Objective: Estimate the sex ratio, age categories, the reproductive status and population size of a colony of $\mathrm{N}$. tumidirostris in this cave. Methodology: The capture of individuals was performed with a handmade harp trap, a modified mist net and manual capture. Results: We capture a total of 274 individuals of which were 14 recaptures. The sex ratio during the samplings was 7: 1 (June), 1: 1 (August) and 3: 1 (September). The structure of the colony was a form of inverted pyramid, and reports the presence of a nursery in April. As for the reproductive status of females, some were inactive ( $42 \%)$ and another $58 \%$ were preparing for reproductive events (proestrus). No reproductive activity was observed in males. The colony size varies in a range of 1941-3279 individuals. Conclusion: For the months of study no sexual segregation occurs, although there were variations in the sex ratio. The presence of a nursery and the description of the reproductive status of females indicate that the breeding season for this species occurs in the first months of the year, between January and February.
\end{abstract}

Keywords: Age category, Chiroptera, Natalidae, Population size, Reproductive status.

* Laboratorio de Ecología Funcional, Unidad de Ecología y Sistemática (UNESIS), Facultad de Ciencias, Pontificia Universidad Javeriana, Bogotá, Colombia. e-mail: analu.045@gmail.com_jaiperez@javeriana.edu.co Fecha recepción: Febrero 4, 2016 Fecha aprobación: Mayo 12, $2016 \quad$ Editor Asociado: Mantilla-Meluk H

(c) Rev. Biodivers. Neotrop. 2016; 6 (2): 227-33 


\section{Introducción}

Natalus tumidirostris Miller, 1900, pertenece a la familia Natalidae (orden Chiroptera), que agrupa murciélagos de tamaño pequeño, con hábitos insectívoros (Tejedor 2011). Los miembros de la familia Natalidae se caracterizan por presentar orejas separadas en forma de embudo, patas largas, cola tan larga como el ancho uropatagio y longitud de la tibia mayor que la mitad de la longitud del antebrazo (Aguirre 2007, Morales-Martínez et al. 2016). Los machos presentan el órgano natálido, que es una masa glandular en el hocico (Morales-Martínez et al. 2016). Natalus tumidirostris se encuentra en Colombia, Venezuela, Guyana, Guyana Francesa, Trinidad y Tobago, Curazao y Bonaire (Dávalos et al. 2008). Para Colombia se reporta en los departamentos de Santander y Córdoba, donde habita únicamente en cuevas que utilizan como refugio y se considera una especie rara y poco estudiada (Tejedor 2011). En la cueva Macaregua en el municipio de Curití, Santander, Colombia $\left(6^{\circ}\right.$ $39^{\prime} 36.2^{\prime \prime} \mathrm{N}$ y $73^{\circ} 0.6^{\prime} 32.3^{\prime}$ " W) se encuentra junto con Desmodus rotundus, Myotis keaysi, Mormoops megalophylla y Carollia perspicillata (Pérez-Torres et al. 2015).

Para N. tumidirostris no se conoce la estructura poblacional, definida por la distribución de edades, proporción de sexos, estado reproductivo y tamaño de la población (Altringham 2011). Aunque esta especie está categorizada por la IUCN como de Baja Preocupación, en el país no existen estudios que indiquen el estado de amenaza en el que se encuentran sus poblaciones (Dávalos et al. 2008). Este trabajo ofrece la primera información sobre estas variables poblacionales en una colonia de esta especie para que sirva de línea base para el análisis de su estado de conservación para el departamento de Santander.

\section{Materiales y métodos}

Area de estudio. El estudio se realizó entre los meses de junio (4 días), agosto (2 días) y septiembre ( 2 días) del año 2013 en la cueva Macaregua (6 $6^{\circ} 39^{\prime}$ $36.2^{\prime \prime} \mathrm{N} \mathrm{y} 73^{\circ} 0.6^{\prime} 32.3$ ' W), ubicada en el municipio de Curití, Santander, que presenta una época seca de noviembre a marzo y una época de lluvia entre abril y octubre (Pérez-Torres et al. 2015).

Métodos. N. tumidirostris fue registrada para la cueva Macaregua en el año 1986 por Gómez-Laverde. Desde el año 2010 investigadores del Laboratorio de Ecología Funcional (LEF) de la Pontificia Universidad Javeriana (PUJ), Bogotá, Colombia, han realizado seguimiento de los murciélagos presentes en ella, y se sabe de la permanencia de la colonia de $N$. tumidirostris en esta cueva (Pérez-Torres et al. 2015). Para capturar la mayor cantidad de individuos posible se combinó el uso de una trampa arpa artesanal, una red de niebla modificada $(135 \times 90 \mathrm{~cm})$ y captura manual directa mientras se encontraban perchados dentro de la cueva. Los muestreos se realizaron durante la mañana entre 6:00 am y10:00 am, momento en el que ya todos los individuos habían regresado a la cueva.

No se realizaron muestreos más exhaustivos debido a las siguientes consideraciones: 1) por el riesgo de contraer histoplasmosis se estableció como protocolo de bioseguridad que el ingreso es con máscaras de filtro y que todo el procesamiento de los individuos se hace fuera de la cueva, 2) como N. tumiditrostris se encuentra dentro de la cueva aproximadamente a $400 \mathrm{~m}$ desde la entrada de esta, se debía asegurar el bienestar de cada individuo minimizando los tiempos de espera desde su captura y procesamiento hasta su liberación (menos de una hora), 3) para asegurar el bienestar de la colonia de $N$. tumidirostris (y las demás especies presentes) se minimizó el número de ingresos y disturbios por el paso y captura de individuos. A cada individuo se le tomaron las medidas morfométricas estándar, el peso, la condición reproductiva y categoría de edad (osificación de la falange) (Kunz 2009). Finalmente, se marcó cada individuo con una tatuadora en el mesopatagio para identificar posibles recapturas y luego calcular el tamaño poblacional.

Se calculó la proporción de sexos por categoría de edad estableciendo la relación entre machos (M) y hembras $(\mathrm{H}): \mathrm{P}=\mathrm{M} / \mathrm{H}$. Adicionalmente, se evaluó la razón de probabilidad de captura entre los machos y hembras mediante una prueba de odds ratio (Bland y Altman 2000).

Para evaluar el estado reproductivo de los machos se tuvo en cuenta el desarrollo y posición de los testículos, considerando individuos inactivos aquellos con testículos impalpables e inguinales y activos los individuos con testículos escrotales. Para el caso de las hembras, se consideró el estado de la sínfisis púbica (abierta, cerrada, intermedia), el estado de la 
vagina (túgida, cornificada, inactiva) y el desarrollo mamario (evidentes, alopécicos, con leche). A todas las hembras se les hicieron además citologías vaginales para determinar en qué etapa del ciclo estral (estro, proestro, anestro o metestro) se encontraban (Vela-Vargas et al. 2016), sin embargo, solo se pudo usar la información del $56 \%$ de las muestras tomadas debido a que en las láminas no se encontró el número de células requeridas para el análisis (Vela-Vargas et al. 2016).

Para establecer las categorías de edad se tuvo en cuenta el grado de osificación de las falanges, las observaciones directas en la guardería encontrada en el mes de abril y la variación en el color del pelaje. Se usaron los siguientes criterios de clasificación de edad: 1) infantiles: individuos de coloración pálida, poco pelaje y osificación incipiente; 2) juveniles: individuos de color gris y comenzando osificación; 3) subadultos: individuos de color café claro con gris y falange osificada incompleta; 4) adultos: individuos de color amarillo oscuro y falange osificada completamente. Con esta información se realizaron las proporciones y porcentajes de individuos capturados en cada categoría de edad de acuerdo con el sexo. Además, se realizó una pirámide poblacional con los datos de todos los muestreos para observar el estado de la población.

Para calcular el tamaño poblacional se utilizaron tres estimadores: media ponderada de Petersen-Lincoln, el método de Schnabel (Schnabel A) y método Schumacher-Eschmeyer (Schnabel B). Estos estimadores asumen que la población es cerrada (donde la natalidad, mortalidad, inmigración y emigración no cambian en el tiempo), que todos los animales tienen la misma probabilidad de captura y que las marcas no se pierden con el tiempo y no afectan a los individuos (Badii et al. 2012). Como cada muestreo mensual tuvo un máximo de cuatro días, para efecto de los análisis, se adoptó la idea que estos supuestos si se cumplen en cada período de muestreo. Finalmente, a los estimadores se les calculó el intervalo de confianza del 95\% con base en la distribución de Poisson (Badii et al. 2012).

\section{Resultados y discusión}

En total se capturaron 274 individuos de los cuales 14 fueron recapturas (260 capturas netas: 73 hembras y 187 machos). La proporción de sexos varió entre meses de muestreo. El número de machos fue mayor en junio (7:1) y septiembre (3:1) y presentó la misma proporción con las hembras en agosto (1:1). A pesar de la variación en las proporciones entre machos y hembras no se evidenció segregación espacial entre ambos sexos. En México, mientras Mitchell (1967) en la región de Sonora no encontró segregación espacial entre machos y hembras para N. mexicanus, Sánchez-Hernández et al. (2002) y Torres-Flores (2013) sí registraron segregación espacial para esta misma especie en Jalisco y Colima. La otra especie insectívora que se encuentra en la cueva de Macaregua (Mormoops megalophylla) sí presenta segregación sexual; las hembras solo están presentes en la cueva durante los meses de junio a septiembre (Delgado 2013).

Durante los pre-muestreos en el mes de abril, se encontró una guardería con aproximadamente 50 crías desnudas, agrupadas en el techo de la cueva y acompañadas por adultos en la periferia (Figura 1). Aunque no se conoce la información sobre el tiempo de gestación de la especie y la duración de las guarderías, de acuerdo con las observaciones directas del presente estudio se puede decir que el tiempo de gestación es de aproximadamente 5 meses y la lactancia alrededor de 2. Las crías de $N$. tumidirostris también nacen desnudas y son dejadas en el techo de los refugios formando grupos con individuos adultos en la orilla constituyendo colonias de maternidad. Las crías de $N$. mexicanus a los siete días tienen su pelaje dorsal gris, a los 15 días este se vuelve más oscuro,

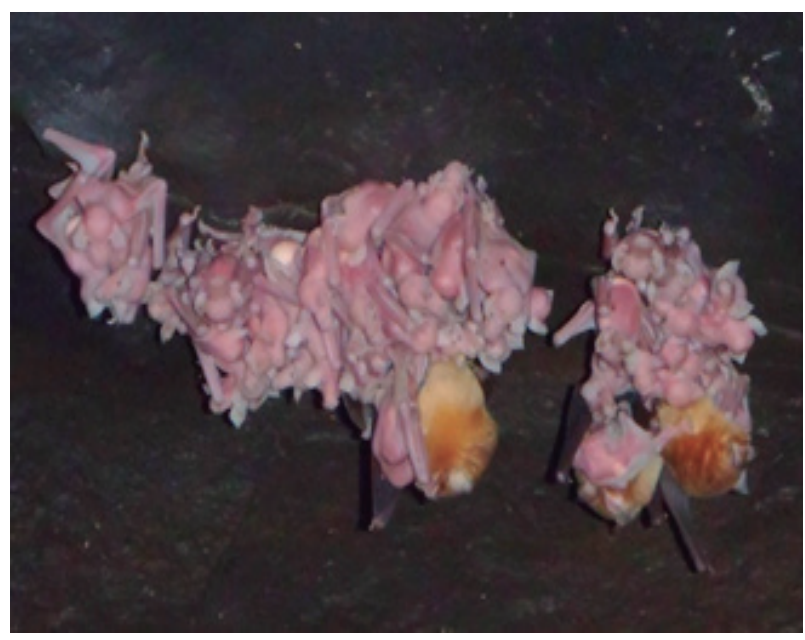

Figura 1. Guardería de Natalus tumidirostris al interior de la cueva Macaregua, Santander, Colombia. 


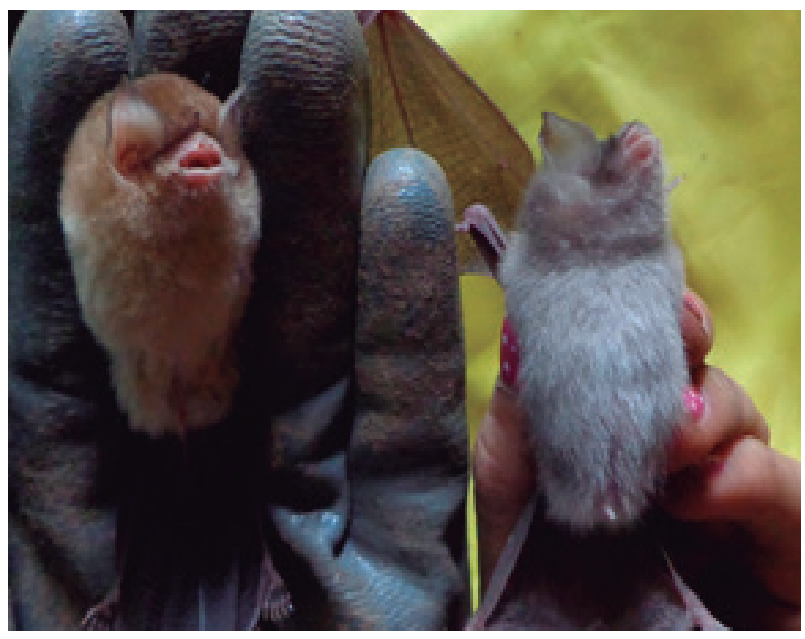

Figura 2. Variación en la coloración de individuos de Natalus tumidirostris. Izquierda: adulto. Derecha: juvenil.

tiempo en el cual las crías comienzan a practicar el vuelo (Sánchez-Hernández et al. 2002). Para el mes de junio también se reporta la presencia de individuos juveniles de color gris y con osificación incompleta de la falange.

De los 260 individuos capturados (excluyendo las 14 recapturas), 176 fueron adultos, 60 subadultos y 24 juveniles. Encontrar una guardería en el mes de abril, permitió registrar mejor las diferencias de color (Figura 2) y el cambio en el grado de osificación para aclarar el criterio de la edad en este estudio, corroborando las características consideradas para $N$. mexicanus por Torres-Flores (2013). Se observó que Natalus tumiditrostris presenta variaciones similares a las reportadas para $N$. mexicanus con individuos juveniles de color gris que a medida que crecen cambian su pelo a coloraciones café y amarillo (Torres-Flores 2013). Gómez-Laverde (1986) aunque no describió una estructura de la colonia sí reportó para la cueva Macaregua una guardería entre los meses de marzo y abril. Se concluye que para la zona de estudio en el departamento de Santander, N. tumidirostris se reproduce en los primeros meses del año (enero y febrero) y las crías nacen durante la época de lluvia entre los meses de marzo y abril. En este período de lluvias la cantidad de insectos es mayor y la actividad reproductiva de los murciélagos insectívoros está determinada por la disponibilidad de insectos (Torres-Flores 2013).

La estructura poblacional presentó una forma de pirámide invertida (Figura 3). Se ha descrito que esta forma de pirámide indica que la colonia tende-

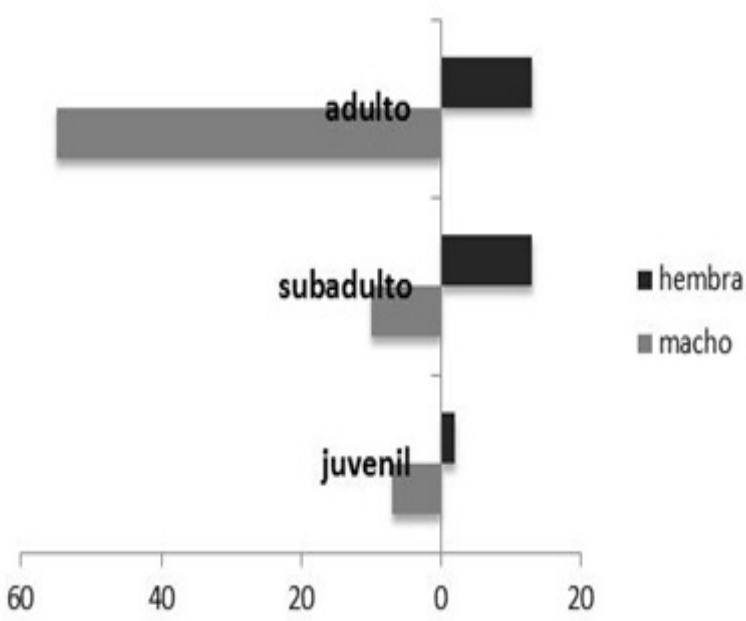

Figura 3. Pirámide poblacional de Natalus tumidirostris en la cueva Macaregua por estado de desarrollo y sexo considerando todos los meses de muestreo.

ría a disminuir al largo plazo porque la cantidad de juveniles es baja en comparación con la de adultos (Rodríguez et al. 2007). No es posible explicar la razón de la forma de la pirámide poblacional que se encontró y tampoco es posible afirmar que realmente la colonia de la cueva está presentando disminución. Sin embargo, sí permite llamar la atención sobre la importancia de seguir evaluando esta misma población para determinar si es un fenómeno continuo y si es así, evaluar qué factores pueden estar determinando este patrón. De esta forma se tendría evidencia específica de riesgo de pérdida de una población local de una especie rara.

La mayoría de las hembras se clasificaron como inactivas de acuerdo con las características externas: vagina inactiva $(95 \%)$, sínfisis púbica cerrada $(87 \%)$, sin pezones evidentes $(77 \%)$ o pezones no alopécicos (89\%). Ninguna se encontraba embarazada o lactante durante el muestreo. Se obtuvieron 41 láminas de citologías vaginales de las hembras que se clasificaron como inactivas. La evaluación del estado reproductivo a partir de las características externas varió con respecto al criterio de las citologías, donde este último es más exacto (Vela et al. 2016). Un poco más de la mitad de estas hembras se encontraron en proestro $(58 \%)$ seguidas de metestro $(35 \%)$ y anestro (7\%), respectivamente. En el proestro se presenta crecimiento de los folículos de Graff, secreción de estrógenos y se preparan para las actividades de cópula que ocurren en la etapa de estro (Nelson 2000). 
Lo anterior muestra que a pesar de clasificarse como inactivas por las características externas, la evidencia de las citologías indica que casi la mitad de las hembras fisiológicamente ya presentan indicios de actividad reproductiva. Por su parte, los machos se presentaron inactivos: la mayoría con los testículos impalpables y en menor proporción inguinales. Sin embargo, siempre se debe tener en cuenta que distinguir la posición de los testículos en esta especie puede ser complicado debido a su pequeño tamaño. Además, se debe considerar que la manipulación de los individuos puede incidir en la posición testicular (Vela 2013). La evidencia encontrada para hembras y machos confirma la descripción de Gómez-Laverde (1986) respecto a que la época reproductiva ocurre en el primer trimestre del año.

De los 274 individuos adultos capturados, se recapturaron 14 (5\%). De acuerdo con los diferentes estimadores empleados, el tamaño de la población varía entre 1941 (IC95\%: 1269-3594) (media ponderada de Peterson-Lincoln), 2080 individuos (IC95\%: 1269-3594) (método A de Schnabel) y 3279 (IC95\%: 1299-8368) (Schumacher y Eschmeyer o Schnabel B).

La semejanza de valores entre los dos primeros se debe a que el método de Schnabel A se basa en la aproximación de Petersen con múltiples eventos de captura (Soriguer y López 1985), mientras que la estimación de Schumacher-Eschmeyer es más robusta y útil ecológicamente ya que utiliza técnicas de regresión a partir del modelo de Schnabel (Freitas-Nery y Marino-Simão 2012, Greenwood et al. 1985). Otros trabajos que también han usado estos estimadores han reportado que el método de Schumacher y Eschmeyer establece un número mayor que el método de Schnabel (Castañeda-Gaytán et al. 2013). Se debe tener en cuenta que el estimador de Schnabel puede subestimar el tamaño poblacional y se considera sesgado (Freitas-Nery y Marino-Simão 2012) debido a que asume igual probabilidad de captura y está negativamente sesgado por la heterogeneidad de las probabilidades de captura (Alexander et al. 1997). De acuerdo con lo anterior, el rango del tamaño de la colonia de $N$. tumidirostris en la cueva Macaregua está entre 1941 y 3279 , siendo poco probable que supere los 3000 individuos.

Natalus tumidirostris también se ha reportado en las cuevas del Nitro y La Alsacia del municipio de
Zapatoca (Santander) y se desconoce aún si se trata de colonias independientes. A diferencia de la cueva Macaregua, en las cuevas del Nitro y La Alsacia se presenta actividad turística constante, sobre todo en la primera (Rueda 2009). Esto en parte debido a que en la cueva Macaregua se ha reportado la presencia del hongo Histoplasma capsulatum, que afecta las vías respiratorias y a que algunas personas ya han sufrido de histoplasmosis luego de visitar la cueva. La cueva Macaregua recientemente fue declarada como Sitio Importante para la Conservación de Murciélagos (SICOM), por la Red Latinoamericana para la Conservación de Murciélagos (RELCOM) por ser la cueva en la que en este momento se registra la mayor cantidad de especies asociadas con una cueva en Colombia, y la presencia de una colonia importante de Natalus tumidirostris. A partir de esta denominación las entidades locales y regionales, manifestaron interés por incluir a la cueva Macaregua dentro de sus listados de áreas de interés a proteger. Es necesario conocer qué está pasando en las demás cuevas de la región del Chicamocha con respecto a las especies que contiene y a los tamaños poblacionales de sus colonias. También es necesario hacer estudios encaminados a determinar si las colonias que se encuentran en diferentes cuevas corresponden a grupos aislados o si por el contario se trata de conjuntos de colonias que presentan intercambio de individuos. También es necesario determinar si el tamaño de la colonia que se reporta en este trabajo es alto o bajo con respecto a otras cuevas de la región y de otras localidades donde se distribuya la especie. Solo así se tendrá un panorama claro del estado de conservación de la especie con miras a una categorización más ajustada de su estatus de conservación.

\section{Conclusiones}

La estimación del tamaño poblacional de $N$. tumidirostris de acuerdo con los estimadores empleados se encuentra en un rango entre 1941 y 3279 individuos, lo cual se constituye en primer estimador poblacional para la especie empleando métodos de captura-recaptura en una cueva de Colombia. Aunque se presentan variaciones en las proporciones entre machos y hembras, no hay un patrón de segregación espacial entre sexos al interior del sistema de cuevas. Aunque una estructura de pirámide invertida sugiere 
una posible tendencia a la declinación poblacional es necesario realizar un seguimiento más detallado para determinar la tendencia numérica de la colonia. La época de apareamientos de esta especie es durante el primer trimestre del año con nacimientos entre marzo y abril.

\section{Agradecimientos}

Al Laboratorio de Ecología Funcional (LEF), Pontificia Universidad Javeriana, por el apoyo logístico y financiero. AE Gómez y F Daza por permitir el acceso a la cueva y colaborar con la logística. AE Delgado, Á Álviz, M Peñuela, Ma T Herrera, M Rodríguez, F Sáenz, I Parra y otros miembros del LEF por su colaboración durante el trabajo de campo y laboratorio. Este trabajo hizo parte del proyecto "Ecología de murciélagos en sistemas cavernícolas en el departamento de Santander" apoyado por la Pontificia Universidad Javeriana (ID 5696).

\section{Literatura citada}

Aguirre LF (ed.). 2007. Historia natural, distribución y conservación de los murciélagos de Bolivia. Santa Cruz: Centro de Ecología y difusión Simón I. Patiño; 400 pp.

Alexander HM, Slade NA, Kettle WD. 1997. Application of mark-recapture models to estimation of the population size of plants. Ecology. 78: 1230-7.

Altringham J. 2011. Bats: From evolution to conservation. $2^{\text {nd }}$ edition. New York: Oxford University Press; 330 pp.

Badii MH, Guillen A, Landeros J, Cerna E, Ochoa Y, Valenzuela J. 2012. Muestreo por métodos de captura-recaptura. Daena: Int J Good Conscience. 7 (1): 97-131.

Bland JM, Altman DG. 2000. Statistics notes. The odds ratio. BMJ. 320 (7247): 1468.

Castañeda-Gaytán G, Gadsden H, López H, Estrada Rodríguez JL. 2003 Historia de vida de Uma paraphygas (Sauria: Phrynosomatiae) en la reserva de la biosfera de Mapimí, Durango. Acta Zool Mex (nueva serie). 89: 169-84.

Dávalos L, Velazco P, Aguirre L. 2008. Natalus tumidirostris. The IUCN Red List of Threatened Species. (En línea). (Acceso junio 26 de 2013). Disponible en: www.iucnredlist.org. http://dx.doi.org/10.2305/IUCN.UK.2008.RLTS. T14362A4435751.en

Delgado E. 2013. Variación de la estructura poblacional de Mormoops megalophylla (Peters, 1864) durante la época húmeda en la cueva Macaregua, Santander, Colombia. (Trabajo de grado). Bogotá: Departamento de Biología, Facultad de Ciencias, Pontificia Universidad Javeriana; 43 pp.

Freitas-Nery M, Marino-Simão S. 2012. Capture-recapture abundance estimate of Guiana dolphins in southeastern Brazil. Ciencias Marinas. 38 (3): 529-41.
Greenwood J, Sargeant B, Johnson H. 1985. Evaluation of mark-recapture for estimating striped skunk abundance. $J$ Wildl Manage. 49 (2): 332-40.

Gómez-Laverde M. 1986. Morfometría y cariología de una población de Natalus tumidirostris (Chiroptera: Natalidae). (Trabajo de Grado). Bogotá: Departamento de Biología, Facultad de Ciencias, Universidad Nacional de Colombia; $70 \mathrm{pp}$.

Kunz T. 2009. Ecological and behavioral methods for the study of bats. $2^{\text {nd }}$ edition. Baltimore: Smithsonian Institution Press; $901 \mathrm{pp}$.

Mejía GC. 2008. Plan de Desarrollo Municipio de Curití 2008-2011. (En línea) (Acceso 10 de julio de 2013). Disponible en: http://www.curiti-santander.gov.co/ apc-aa-files/30363033353966343137363663366231/ PLAN_DE_DESARROLLO_CURIT_1.pdf

Mitchell GC. $196 \overline{7}$. Population study of the funnel-eared bat ( $\mathrm{Na}$ talus stramineus) in Sonora. The Southwestern Naturalist. 12 (2): 172-5. doi: 10.2307/3669271

Morales-Martínez DM, Cárdenas-González C, Fernández-Rodríguez C, Rodríguez-Posada ME, Moreno-Bejarano LM. 2016. Nuevos registros de Natalus tumidirostris (Chiroptera: Natalidae) en Colombia, con notas sobre su variación morfométrica. Mastozool Neotrop. 23 (2). En prensa.

Nelson RJ. 2000. An introduction to behavioral endocrinology. $2^{\text {nd }}$ edition. Baltimore and London: Sinauer Press; $287 \mathrm{pp}$.

Pérez-Torres J, Martínez-Medina D, Peñuela-Salgado M, Ríos-Blanco MC, Estrada-Villegas S, Martínez-Luque L. 2015. Macaregua: the cave with the highest bat richness in Colombia. Check List. 11 (2): 1-6. doi: http://dx.doi. org/10.15560/11.2.1616

Rodríguez A, Álvarez L, Castañeda I. 2007. La pirámide de población. Precisiones para su utilización. Rev Cubana Salud Pública. 33 (4): 1-9.

Rueda C. 2009. Dieta de Carollia perspicillata y Artibeus lituratus asociados a las cuevas El Nitro y La Alsacia (Zapatoca, Santander). (Trabajo de grado). Bogotá: Departamento de Ecología, Facultad de Estudios Ambientales y Rurales, Pontificia Universidad Javeriana; 94 pp.

Sánchez-Hernández C, Romero-Almaraz ML, Gurrola-Hidalgo MA. 2002. Natalus stramineus saturatus (Dalquest y Hall, 1949). Pp. 403-5. En: Noguera FA, Vega Rivera JH, García-Aldrete AN, Quesada M (eds.). Historia Natural de Chamela. México, DF: Instituto de Biología, Universidad Nacional Autónoma de México; 568 pp.

Soriguer RC, López M. 1985. Estudio de una población rural de ratones (Mus musculus L.) II. Análisis comparativo de once estimadores del tamaño poblacional. Doñana Acta Vertebrata. 12 (1): 141-51.

Tejedor A. 2011. Systematics of funnel-eared bats (Chiroptera: Natalidae). Bull Am Museum Nat Hist. 353: 1-140.

Torres-Flores JW. 2013. Dinámica poblacional, patrón reproductivo, dieta, selección de condiciones microclimáticas y hábitos de percha de Natalus mexicanus (Chiroptera: Natalidae) en la parte central de Colima, México. (PhD Tesis). México: Universidad Autónoma Metropolitana; 202 pp.

Vela-Vargas M. 2013. Variaciones en la fenología reproductiva de las especies de murciélagos en dos sistemas ganade- 
ros: Efecto de la disponibilidad de recursos. (Tesis MSc). Bogotá: Pontificia Universidad Javeriana; 89 pp.

Vela-Vargas M, Pérez-Pabón L, Larraín P, Pérez-Torres J. 2016.
Vaginal smears: A key source of information on the estrous cycle of neotropical bats. Mastozool Neotrop. 23 (1): $139-45$. 